\title{
Cambios en hábitos saludables relacionados con actividad física y sedentarismo durante un confinamiento nacional por covid-19 \\ Changes in healthy habits regarding physical activity and sedentary lifestyle during a national lockdown due to covid-19
}

*Eva Santos-Miranda, **Javier Rico-Díaz, **Aida Carballo-Fazanes, **Cristian Abelairas-Gómez *Servicio Galego de Saúde (España); **Universidade de Santiago de Compostela (España)

Resumen. El presente estudio analizó el efecto del confinamiento en España derivado de la COVID-19 en niveles de actividad física diaria, ejercicio físico y comportamientos sedentarios. Tras la declaración del estado de alarma, se difundió un cuestionario por redes sociales con preguntas sociodemográficas, actividad física y comportamientos sedentarios. Posteriormente, se envió por correo electrónico otro cuestionario en relación con la nueva situación de confinamiento. Respondieron a ambos cuestionarios 474 participantes. Se registró un aumento del peso e índice de masa corporal durante el confinamiento $(p<.001)$. Aumentó el número de días en los que se realizaba ejercicio físico en tiempo de ocio $(p<.001)$, pero no el tiempo de práctica semanal total. Se redujo el tiempo de actividad física durante actividades académicas y/o profesionales y el tiempo destinado a desplazamientos $(p<.001)$. Se registró un mayor tiempo de sueño, siestas y sedestación $(p<.01)$. El presente estudio evidencia la necesidad de crear estrategias eficaces encaminadas a aumentar la práctica de actividad física y promover los hábitos de vida saludables, especialmente durante la actual crisis humanitaria y posibles futuras. Palabras clave: ejercicio físico; condición física; hábitos saludables; comportamientos sedentarios; SARS-CoV-2; cuestionarios.

\begin{abstract}
The aim of this study was to analyze the effect of a lockdown in Spain due to COVID-19 on the levels of physical activity, exercise and sedentary lifestyle. After declaring state of alarm in Spain, a questionnaire was distributed via social networks with demographic questions, academic and/or employment status, physical activity and sedentary lifestyle. Subsequently, another questionnaire was sent by email in relation to the new lockdown measures. 474 participants replied to both questionnaires. An increase in weight and body mass index was recorded during lockdown ( $<<0.001)$. The number of days in which physical activity was practiced during leisure time increased $(\mathrm{p}<0.001)$, but the total time of weekly practice stayed the same. The time of physical activity during academic time and the time spent walking was reduced, as well as was the time spent walking $(\mathrm{p}<0.001)$. Longer sleep, naps and sitting time was also recorded $(\mathrm{p}<0.01)$. The present study shows the need to create effective strategies aimed to increase the practice of physical activity and promote a healthy lifestyle during the current humanitarian crisis and possible future ones.
\end{abstract}

Keywords: physical exercise; physical fitness; health behavior; sedentary behavior; SARS-CoV-2, surveys and questionnaires.

\section{Introducción}

En diciembre de 2019, la aparición del síndrome respiratorio agudo grave por coronavirus 2 desencadenó una emergencia sanitaria que provocó, el 31 de enero de 2020, que la Organización Mundial de la Salud (OMS) declarase el brote de este nuevo coronavirus, responsable de la infección llamada COVID-19, una emergencia de salud pública a nivel mundial (Yousfi, et al., 2020), la cual fue catalogada como pandemia mundial el 11 de marzo de 2020 (Organización Mundial de la Salud, 2021). Desde entonces, muchos son los países

Fecha recepción: 12-05-21. Fecha de aceptación: 19-07-21

Cristian Abelairas-Gómez

cristianabelairasgomez@gmail.com azotados por los efectos de esta crisis sanitaria, en datos tanto de morbilidad como de mortalidad. En España, se han notificado más de 4.000.000 de casos de COVID19 y más de 81.000 fallecidos, aunque puede haber discrepancias en las estadísticas resultado de la validación de los datos por las comunidades autónomas (Centro de Coordinación de Alertas y Emergencias Sanitarias, 2021).

Con vistas a limitar la transmisión de la enfermedad, países enteros establecieron confinamientos domiciliarios de la población. En el caso de España, el Real Decreto 463/2020, de 14 de marzo, por el que se declara el estado de alarma para la gestión de la situación de crisis sanitaria ocasionada por la COVID-19, supuso el confinamiento de la población durante varias semanas (Ministerio de la Presidencia, Relaciones con las Cortes y Memoria Democrática, 2020). Además de las re- 
percusiones económicas derivadas de esta situación, especialmente en la población más vulnerable (CastañoRuiz, et al, 2020), muchos son los estudios publicados sobre el efecto a corto y medio plazo de este confinamiento en la salud de la población y en los cambios en su estilo de vida, entre los que resalta el aumento del sedentarismo (Biviá-Roig, et al., 2020; CastañedaBabarro, et al, 2020)' cambios en los hábitos de sueño, aumento del uso de pantallas, enfermedades mentales y estrés (Jurak, et al., 2020; Narici, et al., 2021)

Estos periodos de inactividad, incluso de pocos días, son suficientes para inducir pérdida muscular, aumento de peso (Bhutani \& Cooper, 2020) y de resistencia a la insulina, disminución de la capacidad aeróbica y aumento del riesgo cardiovascular (Biviá-Roig, et al., 2020; Martinez-Ferran, et al., 2020; Pecanha, et al., 2020). Además, la alteración de las rutinas diarias, situación laboral, reuniones sociales previas a la pandemia y momentos de ocio relativamente fijos, combinadas con una exposición reducida a la luz del día, aumenta el riesgo de sufrir problemas de higiene del sueño y alteración de los ritmos circadianos (Morin, et al, 2020). Por tanto, aunque se ha mostrado el confinamiento domiciliario como una de las medidas más efectivas para combatir la transmisión de la enfermedad, también supone un factor de riesgo para la salud de la población. Es por ello que se han estudiado medidas y recomendaciones destinadas a la población confinada, incidiendo en la necesidad de practicar ejercicio físico controlado, diario y adaptado a la nueva situación (Alarcón \& Hall-López, 2020; Bentlage, et al., 2020; García-Tascón, et al., 2020; LópezBueno, Calatayud, Casaña, et al., 2020; Schwendinger \& Pocecco, 2020).

En este sentido, se hace necesario establecer líneas de actuación que nos puedan orientar a luchar contra la inactividad física y sedentarismo durante posibles confinamientos futuros (Alomari, et al., 2020; Colley, et al, 2020; Jurak, et al., 2020). Nuestra hipótesis es que el confinamiento repercute negativamente en los niveles de actividad física aumentando las actividades sedentarias, por lo que el objetivo principal de la presente investigación ha sido estudiar el efecto del confinamiento derivado del Real Decreto 463/2020 (Ministerio de la Presidencia, Relaciones con las Cortes y Memoria Democrática, 2020) en los niveles de actividad física y comportamientos sedentarios, teniendo en cuenta las variables antropométricas, el análisis de la actividad física durante la jornada laboral y ejercicio físico en tiempo de ocio y el posible cambio de tendencias en la práctica habitual de ejercicio físico.

\section{Material y métodos}

\section{Diseño}

Investigación de corte transversal en la que se elaboraron dos cuestionarios online estructurados que interrogaban sobre la situación laboral/académica de los participantes, así como de hábitos relacionados con actividad física, ejercicio físico y comportamientos sedentarios. La principal diferencia entre los dos cuestionarios fue que uno de ellos se refería a la situación anterior a la publicación del Real Decreto 463/2020, de 14 de marzo, por el que se declara el estado de alarma para la gestión de la situación de crisis sanitaria ocasionada por la COVID-19 (Ministerio de la Presidencia, Relaciones con las Cortes y Memoria Democrática, 2020) (en adelante Pre-EA); por su parte, el segundo cuestionario preguntaba por la situación y hábitos posteriores a la publicación de mencionado Real Decreto 463/2020 (en adelante Post-EA).

El cuestionario Pre-EA se difundió a través de redes sociales (Twitter y Facebook) el día 23 de marzo de 2020, que estuvo abierto dos semanas. Se informó a los encuestados de la finalidad del estudio y del tratamiento de datos. Cada participante tenía que incluir una dirección de correo electrónico que sería utilizada para, posteriormente, enviarle el cuestionario Post-EA. Aunque el primer cuestionario fue enviado en un contexto de restricciones máximas y confinamiento domiciliario, las preguntas hacían referencia a una semana tipo previa al establecimiento del estado de alarma, es decir, cuando la práctica deportiva en exteriores todavía estaba permitida y el confinamiento domiciliario todavía no estaba instaurado. El segundo cuestionario fue enviado cuando aún era obligatorio el confinamiento domiciliario y las preguntas trataban de describir los niveles de actividad física y práctica de ejercicio en un contexto de confinamiento domiciliario.

Se siguieron los principios éticos de la Declaración de Helsinki. No se precisó aprobación de comité de ética ya que no se realizó intervención con seres humanos o animales, no se usaron datos de pacientes ni muestras biológicas.

\section{Instrumento}

El formato de ambos cuestionarios (el Pre-EA y el Post-EA) era similar en cuanto a su división por bloques, (demográficos, situación académica/laboral, niveles de actividad física, comportamientos sedentarios), pero las preguntas variaban en cada uno adecuándose a la situación epidemiológica para posteriormente com- 
parar los datos obtenidos (Tabla 1). Estaban constituidos con preguntas de respuesta única y múltiple, además de combinar las de respuesta cerrada y abierta. Todas las preguntas eran de obligada respuesta.

\begin{tabular}{|c|c|c|}
\hline & Pre-EA* & Post-EA \\
\hline \multirow{5}{*}{$\begin{array}{l}\text { Datos socio- } \\
\text { demográficos }\end{array}$} & Sexo & \\
\hline & Edad & \\
\hline & CCAA & \\
\hline & Estado Civil & \\
\hline & Hijos & \\
\hline \multirow{6}{*}{$\begin{array}{c}\text { Datos } \\
\text { antropométricos }\end{array}$} & Altura & \\
\hline & Peso & Peso \\
\hline & IMC & IMC \\
\hline & Estudios & Situación laboral \\
\hline & Titulación & ERTE \\
\hline & $\begin{array}{l}\text { Situación laboral } \\
\text { Jornada laboral }\end{array}$ & Presencialidad/Teletrabajo \\
\hline \multirow{8}{*}{ Actividad física } & Tiempo de EF** en tiempo de ocio al día & \\
\hline & Días de EF a la semana & Tiempo de AF en el trabajo \\
\hline & Tipo de EF & Días de EF a la semana \\
\hline & Compañía & Tipo de EF \\
\hline & Localización & Compañía \\
\hline & Domicilio & Material utilizado \\
\hline & Material utilizado & Tiempo invertido en desplazamientos \\
\hline & $\begin{array}{l}\text { Tiempo invertido en desplazamientos } \\
\text { caminando }\end{array}$ & caminando \\
\hline \multirow{3}{*}{$\begin{array}{l}\text { Sedestación y } \\
\text { sueño }\end{array}$} & Tiempo de sedestación & Tiempo de sedestación \\
\hline & Horas de sueño & Horas de sueño \\
\hline & Horas de siesta & Horas de siesta \\
\hline
\end{tabular}

\section{Análisis estadístico}

Los datos de los cuestionarios fueron importados a una base (Microsoft Excel para Mac, v 16.32) y luego transferidos al programa estadístico SPSS (IBM corp., v. 23.0.0.0) para su análisis. Los datos se expresan en medianas (rango intercuartílico) y frecuencias absolutas (frecuencias relativas) en función de la tipología de cada variable. Para la comparativa entre los cuestionarios Pre-EA y Post-EA se utilizaron el Wilcoxon signedrank test para el análisis de variables continuas y el test de McNemar para el análisis de variables categóricas dicotómicas. Se estableció un nivel de significación de $p$ $<.05$ para todos los análisis.

\section{Resultados}

Se recibieron 1003 respuestas (muestra de conveniencia) del cuestionario Pre-EA y 494 del Post-EA.Tras cotejar los correos electrónicos de las respuestas de ambos cuestionarios, se encontraron 474 pares de res-

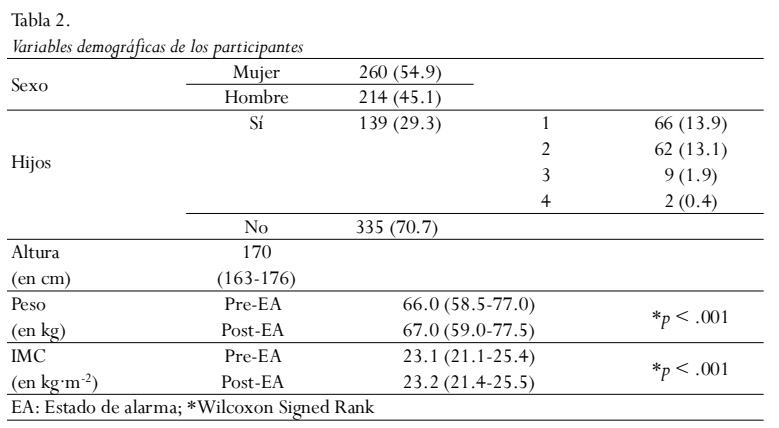

puestas que conformaron la muestra final del presente estudio. El estudio contó con una participación de 214 (45.1\%) mujeres. Se encontró un aumento significativo en el peso e IMC en Post-EA [Peso: 67.0 (59.0-77.5); IMC: 23.2 (21.4-25.5)] respecto a Pre-EA [Peso: 66.0 (58.5-77.0); IMC: 23.1 (21.1-25.4)] ( $p<.001$ en ambos casos) (Tabla 2).

La tabla 3 muestra la situación académica y/o laboral de los participantes, con 254 (53.6\%) estudiantes, de los que 210 (82.7\%) recibían enseñanzas presenciales. En cuanto a situación laboral, 295 (62.2\%) afirmaron trabajar en Pre-EA, cifra que se redujo en Post-EA [211 (44.5\%), $p<.001]$. Estos datos fueron útiles para analizar los niveles de actividad física relacionados con la ocupación antes y después del establecimiento del estado de alarma.

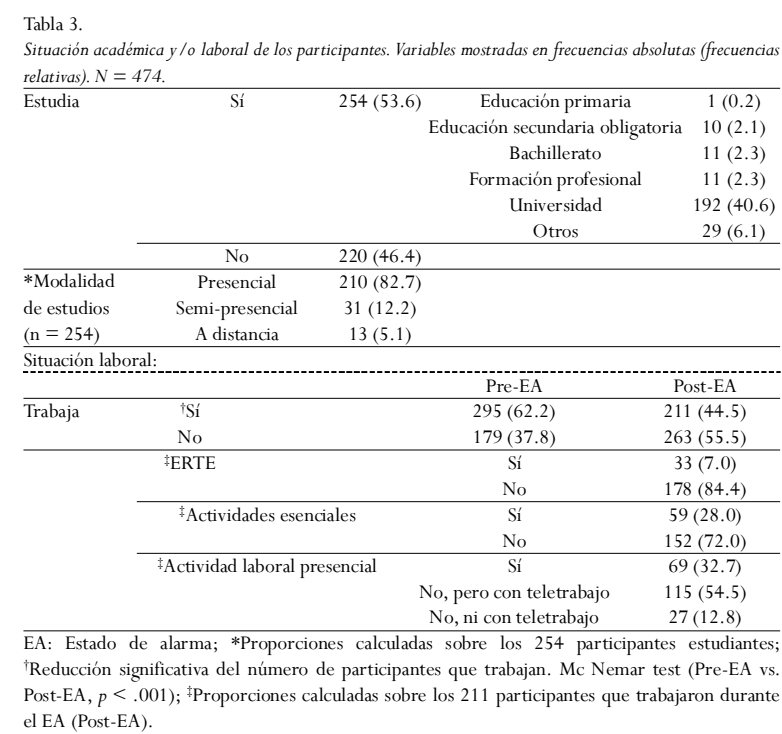

Comparando los niveles de actividad física a lo largo de la jornada académica y/o laboral, en Post-EA se observó un incremento en el número de participantes que no realizaba ningún tipo de actividad física (Figura 1). También en Post-EA ha descendido el porcentaje de participantes cuyas jornadas laborales suponían al menos un 25\% de actividad física.

En referencia a la práctica de ejercicio físico en tiempo de ocio, 447 (94.3\%) encuestados afirmaron realizar

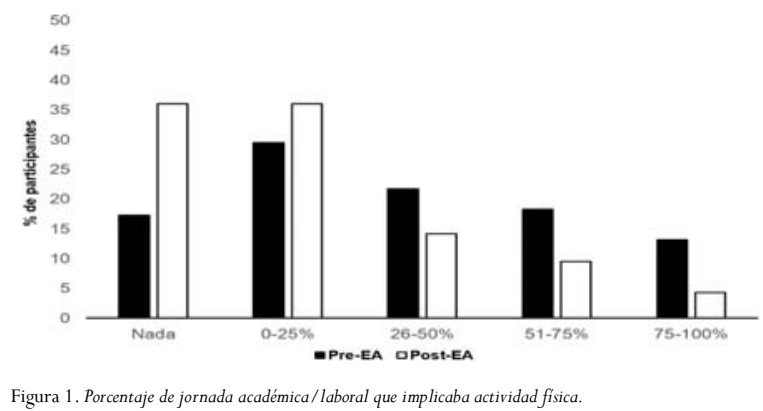


Tabla 4.

Desplazamientos y ejercicio físico.

\begin{tabular}{ccccc}
\hline & & Pre-EA & Post-EA & $P$ \\
\hline \multicolumn{2}{c}{ Días de ejercicio físico semanales } & $4(3-6)$ & $5(4-7)$ & $<.001^{\dagger}$ \\
\hline \multicolumn{2}{c}{ Tiempo semanal de ejercicio físico (min) } & 240 & 240 & $.9366^{\dagger}$ \\
\hline \multirow{2}{*}{ Actividad física en casa } & Sí & $140-420)$ & $(120-420)$ & \\
\hline \multirow{5}{*}{ *Desplazamientos semanales } & No & $331(69.8)$ & $448(94.5)$ & $<.001^{\ddagger}$ \\
(min) & $<10$ & $164(34.6)$ & $355(74.9)$ & \\
& $10-60$ & $120(25.3)$ & $76(16.0)$ & \\
& $61-120$ & $91(19.2)$ & $27(5.7)$ & $<.001^{\ddagger}$ \\
& $121-180$ & $40(8.4)$ & $3(0.6)$ & \\
EA: Estado de alarma; AF: Actividad física; *Solo se contabilizan desplazamientos de al menos
\end{tabular}

10 min de duración ${ }^{\dagger}$; Wilcoxon signed-rank test: ${ }^{\ddagger}$. McNemar tost.

Tabla 5.

Con quién y dónde se realizaba actividad fisica antes y después del decreto del estado de alarma. Variables mostradas en frecuencias absolutas (frecuencias relativas).

\begin{tabular}{|c|c|c|c|c|c|}
\hline & & & $\begin{array}{l}\begin{array}{l}\text { Pre-EA } \\
(\mathrm{n}=447)\end{array} \\
\end{array}$ & $\begin{array}{l}\text { Post-EA } \\
(\mathrm{n}=448)\end{array}$ & $\begin{array}{c}\text { McNemar } \\
\mathrm{p}\end{array}$ \\
\hline \multirow[t]{11}{*}{ Con quién } & \multicolumn{2}{|c|}{ Solo/a } & $282(63.1)$ & $339(75.7)$ & $<0.001$ \\
\hline & \multirow{2}{*}{ Amigos/as } & Pres & $149(33.3)$ & $7(1.6)$ & $<0,001$ \\
\hline & & Tele & -...- & $51(11.4)$ & $<0,001$ \\
\hline & \multirow{2}{*}{ Familia } & Pres & $44(9.8)$ & $136(30.4)$ & \\
\hline & & Tele & -..- & $11(2.5)$ & $<0.001$ \\
\hline & \multirow{2}{*}{ Pareja } & Pres & $52(11.6)$ & $81(18.1)$ & $<0,001$ \\
\hline & & Tele & -...- & $21(4.7)$ & \\
\hline & \multirow{2}{*}{ Equipo } & Pres & $157(35.1)$ & $\ldots$ & \\
\hline & & Tele & -...- & $42(9.4)$ & $<0.001$ \\
\hline & \multirow{2}{*}{ Con entrenador $/ \mathrm{a}$} & Pres & $1(0.2)$ & $\cdots$ & 0063 \\
\hline & & Tele & -..-- & $6(1.3)$ & \\
\hline \multirow[t]{13}{*}{ *Lugar } & \multicolumn{2}{|c|}{ En un centro deportivo/gimnasio } & $274(61.3)$ & & \\
\hline & \multirow{2}{*}{\multicolumn{2}{|c|}{$\begin{array}{l}\mathrm{Al} \text { aire libre } \\
\text { En mi casa }\end{array}$}} & $252(56.4)$ & & \\
\hline & & & $143(32.0)$ & $448(94.5)$ & \\
\hline & \multicolumn{2}{|c|}{ En un club deportivo } & $129(28.9)$ & & \\
\hline & \multicolumn{2}{|c|}{ Estudio de pilates/yoga } & $4(0.9)$ & & \\
\hline & \multicolumn{2}{|c|}{ Pistas: padel/tenis/atletismo } & $3(0.7)$ & & \\
\hline & \multicolumn{2}{|c|}{ CTD } & $2(0.4)$ & & \\
\hline & \multicolumn{2}{|c|}{ Escuela de baile/danza } & $2(0.4)$ & & \\
\hline & \multicolumn{2}{|c|}{ En la facultad } & $2(0.4)$ & & \\
\hline & \multicolumn{2}{|c|}{ Centro cultural } & $2(0.4)$ & & \\
\hline & \multicolumn{2}{|c|}{ Academia } & $1(0.2)$ & & \\
\hline & \multicolumn{2}{|c|}{ Servicio de Deportes de la Universidad } & $1(0.2)$ & & \\
\hline & & & $1(0.2)$ & & \\
\hline
\end{tabular}

Telemático. *Datos solamente descriptivos.

\section{Tabla 6.}

Actividad fisica en casa y material disponible en el hogar para la práctica de actividad fisica. Variables mostradas en frecuencias absolutas (frecuencias relativas).

\begin{tabular}{|c|c|c|c|c|}
\hline & & Pre-EA & Post-EA & McNemar \\
\hline \multirow{7}{*}{$\begin{array}{c}\text { Realiza } \\
\text { actividad } \\
\text { física en } \\
\text { casa }\end{array}$} & Sí & $143(32.0)$ & $448(94.5)$ & $<0.001$ \\
\hline & Tutoriales/masterclass a través de plataformas & 48 (33.6) & $282(62.9)$ & $<0.001$ \\
\hline & Trabajo de fuerza con material & $95(66.4)$ & $276(61.6)$ & 0.345 \\
\hline & Trabajo de fuerza sin material & 105 (73.4) & $345(77.0)$ & 0.003 \\
\hline & Cardio: correr/ bici/ elíptica... & $51(35.7)$ & $188(42.0)$ & $<0.001$ \\
\hline & Cardio: circuito de ejercicios & $56(39.2)$ & $273(60.9)$ & $<0.001$ \\
\hline & Yoga/ pilates... & $42(29.4)$ & $163(36.4)$ & 0.018 \\
\hline \multirow{15}{*}{$\begin{array}{l}* \text { Materia } \\
\text { l para } \\
\text { realizar } \\
\text { actividad } \\
\text { física en } \\
\text { casa }\end{array}$} & Sí & $302(63.7)$ & $342(72.2)$ & 0.001 \\
\hline & Gomas elásticas & $212(70.4)$ & $221(64.6)$ & \\
\hline & Tapiz rodante, bici estática o similar & $105(34.9)$ & $144(42.1)$ & \\
\hline & Pesas & $101(33.6)$ & $158(46.2)$ & \\
\hline & Esterilla/Ladrillos & $5(1.7)$ & $10(2.9)$ & \\
\hline & Barra de dominadas & $3(1.0)$ & $3(0.9)$ & \\
\hline & Fitball & $2(0.7)$ & $4(1.2)$ & \\
\hline & TRX & $2(0.7)$ & $7(2.0)$ & \\
\hline & Saco de boxeo & $1(0.3)$ & $1(0.3)$ & \\
\hline & Comba & $1(0.3)$ & $3(0.9)$ & \\
\hline & Bosu & $1(0.3)$ & $2(0.6)$ & \\
\hline & Anillas & $1(0.3)$ & $2(0.6)$ & \\
\hline & Balón medicinal & $1(0.3)$ & $2(0.6)$ & \\
\hline & Foam roller & -. & $1(0.3)$ & \\
\hline & Escalera de coordinación & -. & $1(0.3)$ & \\
\hline
\end{tabular}

\section{EA: Estado de alarma}

Escalera de coordinació

*Solamente es analizaron diferencias entre grupos en la variable referida a poseer material para realizar actividad física en casa, no para cada material.

ejercicio físico en Pre-EA y 448 (94.5\%) en Post-EA. Los participantes afirmaron ejercitarse en sus tiempos de ocio durante más días a la semana en Post-EA [5 (47) días] que en Pre-EA [4 (3-6) días] $(p<.001)$; sin embargo, no se ha observado un aumento significativo en el tiempo semanal de ejercicio físico medido en mi- nutos (Tabla 4). El número de participantes que realizaba ejercicio físico en casa aumentó significativamente en Post-EA, así como el número de participantes que no alcanzó desplazamientos semanales de al menos 10 min continuos ( $p<.001$ en ambos casos).

En las tablas 4-5 se muestran los cambios de tendencias y rutinas en cuanto a práctica de ejercicio físico. En Post-EA, aumentó significativamente el porcentaje de participantes que se ejercitaba en solitario, y aquellos que lo hacían con familiares (Tabla 5) ( $p<.001$ en ambos casos).

En Post-EA aumentó significativamente el uso de tutoriales a través de plataformas virtuales para la práctica de ejercicio físico $(p<.001)$; también se vio incrementado el número de participantes que afirmó poseer material para la práctica de ejercicio físico en casa $(p<.001)$ (Tabla 6). Dentro del tipo de ejercicio realizado por los participantes en Pre-EA, fue el trabajo de fuerza el realizado por más participantes [267 (59.7\%)] (Tabla 7)

\begin{tabular}{|c|c|c|}
\hline & fi & hi \\
\hline Trabajo de fuerza con pesas, gomas, con el propio peso del cuerpo, etc... & 267 & 59.7 \\
\hline Correr & 183 & 40.9 \\
\hline Caminar (no cuentan desplazamientos) & 127 & 28.4 \\
\hline Deportes colectivos & 92 & 20.6 \\
\hline Bicicleta & 91 & 20.4 \\
\hline Nadar & 72 & 16.1 \\
\hline Deportes individuales & 54 & 12.1 \\
\hline Zumba, aeróbic, body combat, etc... & 54 & 12.1 \\
\hline Deportes de raqueta & 38 & 8.5 \\
\hline Baile o danza & 36 & 8.1 \\
\hline Deportes de combate & 27 & 6.0 \\
\hline Pilates & 17 & 3.8 \\
\hline Yoga & 15 & 3.4 \\
\hline Piragüismo & 4 & 0.9 \\
\hline Escalada & 3 & 0.7 \\
\hline Carreras de obstáculos & 2 & 0.4 \\
\hline Crossfit & 2 & 0.4 \\
\hline Kayak & 2 & 0.4 \\
\hline Patinaje & 2 & 0.4 \\
\hline Skate & 2 & 0.4 \\
\hline Acrobacias & 1 & 0.2 \\
\hline Atletismo & 1 & 0.2 \\
\hline Automobilismo & 1 & 0.2 \\
\hline Esquí & 1 & 0.2 \\
\hline Hipopresivos & 1 & 0.2 \\
\hline Remo & 1 & 0.2 \\
\hline Treking & 1 & 0.2 \\
\hline
\end{tabular}

Con respecto a los comportamientos sedentarios, se observó un aumento significativo en Post-EA del tiempo de sedestación, sueño y siesta $(p<.01$ en todos los casos) (Tabla 8).

Tabla 8 .

Comportamientos sedentarios. Variables descritas por mediana (rango intercuartílico) a excepción de Siesta descrita mediante frecuencia absoluta (relativa). $N=474$.

\begin{tabular}{|c|c|c|c|c|}
\hline & & Pre-EA & Post-EA & $\mathrm{p}$ \\
\hline & Sedestación por día (en h) & $6(4-8)$ & $8(5-10)$ & $<0.001 *$ \\
\hline & Sueño $(\mathrm{en} \mathrm{h})$ & $7(7-8)$ & $8(8-9)$ & $<0.001 *$ \\
\hline \multirow{5}{*}{ Siesta } & $\mathrm{Si}$ & $115(24.3)$ & $168(35.4)$ & \multirow{2}{*}{$<0.001^{\dagger}$} \\
\hline & No & $359(75.7)$ & $306(64.6)$ & \\
\hline & Días de siesta por semana & $4(3-5)$ & $4(3-5)$ & $0.051 *$ \\
\hline & Duración de cada siesta (en min) & $30(30-60)$ & $40(30-60)$ & $0.034 *$ \\
\hline & Tiempo de siesta por semana (en min) & $120(90-240)$ & $140(100-240)$ & $0.008 *$ \\
\hline
\end{tabular}

EA: Estado de alarma; *Wilcoxon Signed-Rank test; ${ }^{\dagger}$ McNemar test. 


\section{Discusión}

Desde el establecimiento del estado de alarma en España en marzo de 2020 muchos han sido los estudios que han analizado el impacto del confinamiento en diferentes hábitos relacionados con la salud como la nutrición (Huber, et al, 2021; Philippe, et al, 2021; Poelman, et al., 2021; Robinson, et al., 2021), la salud mental (Carriedo, et al, 2020; Fiorenzato, et al., 2021), o la práctica de ejercicio físico (Carriedo, et al, 2020; Robinson, et al., 2021).

Los resultados de nuestro estudio muestran un cambio significativo en los hábitos de actividad física y comportamientos sedentarios de los participantes durante el confinamiento domiciliario. Se ha observado un descenso de actividad física durante el confinamiento, especialmente en aquella relacionada con la actividad académica/laboral y los desplazamientos. Además, se ha confirmado un aumento significativo del tiempo de sedentarismo. El impacto del confinamiento ha influido también en las condiciones de realización de ejercicio físico y en el uso generalizado de material complementario.

En nuestro estudio no ha variado el número de participantes que realizaba ejercicio en tiempo de ocio antes y durante el confinamiento; de hecho, ha aumentado el número de días semanales que practicaban ejercicio físico. Sin embargo, al no verse modificado el tiempo total semanal de práctica en tiempo de ocio, pero haberse reducido significativamente el porcentaje de jornada laboral/académica que requería actividad física, y al haber descendido también drásticamente el tiempo destinado a desplazamientos, puede afirmarse que el tiempo total de actividad física de los participantes encuestados durante el confinamiento se ha reducido.

Este descenso en los niveles de actividad física supone un perjuicio para la salud desde una doble perspectiva: por un lado, desde la OMS ya se ha alertado de los bajos índices de actividad física por parte de la población general (DiPietro, et al., 2020), informando en 2018 que en España menos del 25\% de adolescentes cumplían con las recomendaciones de práctica de ejercicio, y menos del 70\% en el caso de los adultos (Organización Mundial de la Salud, 2018). Un estudio reciente realizado en España con población mayor ( $>60$ años) reveló que, durante las semanas de confinamiento, el cumplimiento de las recomendaciones de la OMS en cuanto a actividad física de intensidad moderada y vigorosa estuvo asociado con niveles más altos de autoeficacia, optimismo y menores síntomas depresivos (Carriedo, et al., 2020). Por ello, durante el estado de pandemia, deberían evitarse los días de inactividad física, y procurar alcanzar al menos 60 min de práctica diaria de intensidad moderada (Jurak, et al., 2020).

Por otro lado, el descenso en los índices de actividad física conlleva un aumento de comportamientos sedentarios y sus riesgos asociados. Los resultados del presente estudio mostraron un aumento del tiempo de sueño, de siestas y sedestación. Aunque el aumento de las horas de sueño diarias no implica ningún aspecto negativo por sí sólo, al agravio para la salud que supone un decremento de los niveles de actividad física, hay que añadirle el agravante de las horas totales de sedentarismo (García-Tascón, et al., 2020; López-Bueno, Calatayud, Casaña, et al., 2020; 2020; Narici, et al., 2021; Pecanha, et al., 2020; Schwendinger \& Pocecco, 2020). Estudios previos también mostraron que, a raíz de las restricciones tomadas tanto a nivel nacional como internacional tras la declaración del estado de pandemia, y especialmente causa de los confinamientos, se ha incrementado el tiempo destinado a actividades sedentarias (AlonsoMartínez, et al., 2021) como el uso de dispositivos electrónico-digitales (Gallè, et al., 2020; Schmidt, et al., 2020) (ordenadores, televisión, teléfonos móviles, etc.).

El tiempo prolongado de estancia en el domicilio, la falta de espacio y las restricciones de movilidad son factores que influyen en el descenso de práctica de ejercicio y aumento de comportamientos sedentarios. Además, todos estos factores en su conjunto aumentan el riesgo de estrés, depresión, ansiedad y deterioro en la calidad del sueño (Alonso-Martínez, et al., 2021; Altena, et al., 2020; Carriedo, et al., 2020; Jurak, et al., 2020; López-Bueno, Caltayud, Ezzatvar, et al., 2020). Investigaciones previas también han mostrado cambios negativos en los hábitos nutricionales de la población en términos de ingesta (Huber, et al., 2021) o calidad de la dieta (Robinson, et al., 2021). En nuestro estudio, aunque no se han analizado hábitos relacionados con la dieta, sí que se han observado ligeros aumentos en el peso e IMC de los participantes, posiblemente derivados de un balance positivo resultado de la disminución de actividad física, aumento de las horas totales de sedentarismo y la posibilidad de que la ingesta calórica se haya visto aumentada.

\section{Limitaciones}

Nuestro estudio no está libre de limitaciones. La principal limitación del estudio supone el uso de un cuestionario para la medición de los niveles de actividad 
física. Además, a pesar de ser un cuestionario anónimo, al considerarse el sedentarismo un tema socialmente indeseable, los participantes podrían caer en el sesgo de complacencia social y alterar sus respuestas de cara a las que socialmente se consideran como buenas o deseables. También está presente el sesgo de selección, ya que la edad media de los encuestados es relativamente joven teniendo en cuenta que el modo de difusión de los cuestionarios son las redes sociales. Finalmente, el número limitado de participantes dificulta la extrapolación de los resultados al resto de la población.

\section{Conclusiones}

El confinamiento ha supuesto un descenso en los índices de actividad física y aumento de comportamientos sedentarios en la muestra encuestada, evidenciando un aumento del peso, de índice de masa corporal y de horas de sueño. El presente estudio evidencia la necesidad de crear estrategias eficaces encaminadas a aumentar los niveles de actividad física, disminuir los comportamientos sedentarios y promover los hábitos de vida saludables con el fin de mantener la salud de la población y prevenir enfermedades asociadas, especialmente durante la actual crisis humanitaria y posible futura.

\section{Referencias}

Alarcón Meza, E., \& Hall-López, J. (2020). Actividad física en estudiantes deportistas universitarios, previo y en el confinamiento por pandemia asociada al COVID-19. Retos, 39, 572-575.

Alomari, M.A.; Khabour, O.F. \& Alzoubi, K.H. (2020). Changes in physical activity and sedentary behavior amid confinement:The bksq-covid-19 project. Risk Management and Healthcare Policy, 13, 1757-1764.

Alonso-Martínez,A.M.; Ramírez-Vélez, R.; García-Alonso, Y.; Izquierdo, M.\& García-Hermoso,A. (2021). Physical Activity, Sedentary Behavior, Sleep and Self-Regulation in Spanish Preschoolers during the COVID-19 Lockdown. International Journal of Environmental Research and Public Health, 18(2), 693.

Altena, E.; Baglioni, C.; Espie, C.A.; Ellis, J.; Gavriloff, D.; Holzinger, B.; ...; Riemann, D. (2020). Dealing with sleep problems during home confinement due to the COVID 19 outbreak: Practical recommendations from a task force of the European CBT I Academy. Journal of Sleep Research, 29(4), e13052.

Bentlage, E.; Ammar, A.; How, D.; Ahmed, M.; Trabelsi, K.; Chtourou, H.; ...; Brach, M. (2020). Practical recommendations for maintaining active lifestyle during the covid-19 pandemic: A systematic literature review. International Journal of Environmental Research and Public Health, 17(17), 6265

Bhutani. S. \& Cooper, J.A. (2020). COVID-19-Related Home Confinement in Adults: Weight Gain Risks and Opportunities. Obesity, 28(9), 1576-1577.

Biviá-Roig, G.; La Rosa, V.L.; Gómez-Tébar, M.; SerranoRaya, L.; Amer-Cuenca, J.J.; Caruso, S.; . . ; Lisón, J.F. (2020). Analysis of the impact of the confinement resulting from covid-19 on the lifestyle and psychological wellbeing of spanish pregnant women:An internet-based cross-sectional survey. International Journal of Environmental Research and Public Health, 17(16), 5933.

Carriedo, A.; Cecchini, J.A.; Fernandez-Rio, J. \& MéndezGiménez, A. (2020). COVID-19, Psychological Wellbeing and Physical Activity Levels in Older Adults During the Nationwide Lockdown in Spain. The American Journal of Geriatric Psychiatry, 28(11), 1146-1155.

Castañeda-Babarro, A.; Coca, A.; Arbillaga-Etxarri, A. \& Gutiérrez-Santamaría, B. (2020). Physical activity change during COVID-19 confinement. International Journal of Environmental Research and Public Health, 17(18), 6878

Castaño-Ruiz, V.; López, J.; Marí, Samuel. (2020). El impacto de la crisis del COVID-19 en la adolescencia en España: análisis de situación, respuesta y recomendaciones. Observatorio de la Infancia en Andalucía. Recuperado de https://www.observatoriodelainfancia.es/ ficherosoia/documentos/7107_d_ImpactoCOVIDAdolescencia.pdf

Centro de Coordinación de Alertas y Emergencias Sanitarias. (2021). Actualización $n^{\circ}$ 412. Enfermedad por coronavirus (COVID-19). Recuperado de https:// www.mscbs.gob.es/profesionales/saludPublica/ccayes/ alertas Actual / n Cov/documentos / Actualizacion_412_COVID-19.pdf [Consulta: julio 2021].

Colley, R.C.; Bushnik, T. \& Langlois, K. (2020). Exercise and screen time during the COVID-19 pandemic. Health Reports, 31(6), 3-11

DiPietro, L.; Al-Ansari, S.S.; Biddle, S.J.H.; Borodulin, K.; Bull, F.C.; Buman, M.P.; ...; Willumsen, J.F. (2020). Advancing the global physical activity agenda: recommendations for future research by the $2020 \mathrm{WHO}$ physical activity and sedentary behavior guidelines development group. International Journal of Behavioral Nutrition and Physical Activity, 17(1), 143.

Fiorenzato, E.; Zabberoni, S.; Costa, A. \& Cona, G. (2021). Cognitive and mental health changes and their vulnerability factors related to COVID-19 lockdown in Italy. PLoS One, 16(1), e0246204.

Gallè, F.; Sabella, E.A.; Ferracuti, S.; De Giglio, O.; 
Caggiano, G.; Protano, C.; ...; Napoli, C. (2020). Sedentary behaviors and physical activity of Italian undergraduate students during lockdown at the time of COVID»19 pandemic. International Journal of Environmental Research and Public Health, 17(17), 6171.

García-Tascón, M.; Sahelices-Pinto, C.; Mendaña-Cuervo, C. \& Magaz-González, A.M. (2020). The impact of the covid-19 confinement on the habits of pa practice according to gender (Male/female): Spanish case. International Journal of Environmental Research and Public Health, 17(19), 6961.

Huber, B.C.; Steffen, J.; Schlichtiger, J. \& Brunner, S. (2021). Altered nutrition behavior during COVID-19 pandemic lockdown in young adults. European Journal of Nutrition, 60,2593-2602..

Jurak, G.; Morrison,S.A.; Leskošek, B.; Kovaè, M.;Had•iæ, V.; Vodièar, J,; ...; Starc, G. (2020). Physical activity recommendations during the coronavirus disease-2019 virus outbreak. Journal of Sport and Health Science, 9(4), 325-327.

López-Bueno, R.; Calatayud, J.; Casaña, J.; Casajús, J.A.; Smith, L.;Tully, M.A.; . .; López, G.F. (2020). COVID19 Confinement and Health Risk Behaviors in Spain. Frontiers in Psychology, 11, 1426.

López-Bueno, R.; Calatayud, J.; Ezzatvar, Y.; Casajús, J.A.; Smith,L.;Andersen, L.L. \& López-Sánchez, G.F.(2020). Association Between Current Physical Activity and Current Perceived Anxiety and Mood in the Initial Phase of COVID-19 Confinement. Frontiers in Psychiatry, 11, 729 .

Martinez-Ferran, M.; de la Guía-Galipienso, F.; SanchisGomar, F.;Pareja-Galeano, H. (2020). Metabolicimpacts of confinement during the COVID-19 pandemic due to modified diet and physical activity habits. Nutrients, 12(6), 1549.

Ministerio de la Presidencia, Relaciones con las Cortes y Memoria Democrática. (2020). Real Decreto463/2020, de 14 de marzo, por el que se declara el estado de alarma para la gestión de la situación de crisis sanitaria ocasionada por el COVID-19, BOE núm. 67, de 14 de marzo $\$ 25390$. Recuperado de: https://www.boe.es/eli/ es/rd/2020/03/14/463/con

Morin CM, Carrier J, Bastien C, Godbout R. (2020). Sleep and circadian rhythm in response to the COVID-19 pandemic. Canadian Journal of Public Health, 111(5), 654 657.

Narici,M;DeVito, G;Franchi, M;Paoli,A;Moro,T;Marcolin G, ..., Maganaris C. (2021). Impact of sedentarism due to the COVID-19 home confinement on neuromuscular, cardiovascular and metabolic health: Physiological and pathophysiological implications and recommendations for physical and nutritional countermeasures. European Journal of Sport Science, 21(4), 614-635.

Organización Mundial de la Salud. (2018). Spain Physical Activity Factsheet 2018. Recuperado de https:// www.euro.who.int/_data/assets/pdf_file/0008/ 382580/spain-eng.pdf.

Organización Mundial de la Salud (2021).Alocución de apertura del Director General de la OMS en la rueda de prensa sobre la COVID-19 celebrada el 11 de marzo de 2020. Recuperado de https:/ / www.who.int/es/director-general/speeches/detail/who-director-general-sopening-remarks-at-the-media-briefing-on-covid-19 11-march-2020

Pecanha,T.; Goessler, K.F.; Roschel, H. \& Gualano, B. (2020). Social isolation during the COVID-19 pandemic can increase physical inactivity and the global burden of cardiovascular disease. American Journal of Physiology-Heart and circulatory Physiology, 318(6), H1441-H1446.

Philippe, K.; Chabanet, C.; Issanchou, S. \& Monnery-Patris, S. (2021). Child eating behaviors, parental feeding practices and food shopping motivations during the COVID-19 lockdown in France: (how) did they change? Appetite, 161, 105132

Poelman, M.P.; Gillebaart, M.; Schlinkert, C.; Dijkstra, S.C.; Derksen, E.; Mensink, F.; ...; de Vet, E. (2021). Eating behavior and food purchases during the COVID19 lockdown: A cross-sectional study among adults in the Netherlands. Appetite, 157, 105002.

Robinson, E.; Boyland, E.; Chisholm, A.; Harrold, J.; Maloney, N.G.; Marty, L.; ...; Hardman, C.A. (2021). Obesity, eating behavior and physical activity during COVID-19 lockdown: A study of UK adults. Appetite, 156,104853

Schmidt, S.C.E.; Anedda, B.; Burchartz, A.; Eichsteller, A.; Kolb, S.; Nigg, C.; . . ; Woll, A. (2020). Physical activity and screen time of children and adolescents before and during the COVID-19 lockdown in Germany: a natural experiment. Scientific Reports, 10(1), 21780.

Schwendinger, F. \& Pocecco, E. (2020). Counteracting physical inactivity during the COVID-19 pandemic: Evidence-based recommendations for home-based exercise. International Journal of Environmental Research and Public Health, 17(11), 3909.

Yousfi, N.; Bragazzi, N.L.; Briki, W.; Zmijewski, P. \& Chamari, K. (2020). The COVID-19 pandemic: How to maintain a healthy immune system during the lockdown - A multidisciplinary approach with special focus on athletes. Biology of Sport, 37, 211-216. 